\section{PTU-061 OUTCOMES OF PAEDIATRIC LIVER TRANSPLANT RECIPIENTS FOLLOWING TRANSITION FROM PAEDIATRIC TO ADULT HEALTHCARE SERVICES}

\section{doi:10.1136/gutjnl-2012-302514c.61}

${ }^{1} \mathrm{~N}$ Sagar, ${ }^{1} \mathrm{~J}$ Leithead, ${ }^{2} \mathrm{D}$ Kelly, ${ }^{1} \mathrm{D}$ Adams, ${ }^{2} \mathrm{~J}$ Arkley, ${ }^{1} \mathrm{~B}$ Gunson, ${ }^{2} \mathrm{P}$ McKiernan, ${ }^{2} \mathrm{M}$ Smith, ${ }^{2} \mathrm{P}$ Lewis, ${ }^{1} \mathrm{~J}$ Ferguson. 'Oueen Elizabeth Hospital Liver Unit, Birmingham, UK; ${ }^{2}$ Birmingham Children's Hospital, Birmingham, UK

Introduction Given the success of paediatric transplantation, there is now a growing population of patients that undergo transition from paediatric to adult healthcare services. In the renal transplant setting, these patients appear to be at increased risk of short-term graft loss. ${ }^{1}$ However, the outcome of liver transplant patients who undergo transition remains unclear. Our aim was, therefore, to examine the patient and graft survival of liver transplant patients who undergo paediatric to adult healthcare transition.

Methods Single-centre retrospective study of 85 liver transplant recipients who underwent transition from paediatric to adult healthcare services between March 2001 and August 2011 (median follow-up time from transition 4.1 (range 0.2-10.7) years).

Results During the 10-year period, there were 48 (56.5\%) males and $37(43.5 \%)$ females. The median age at time of transplant was 8.4 (range 0.6-17.9) years. The initial indications for transplantation were: biliary atresia (27.1\%), Wilson's $(14.1 \%)$, other acute liver failure (11.8\%), cystic fibrosis $(9.4 \%)$, autoimmune hepatitis $(8.2 \%)$, cryptogenic cirrhosis $(4.7 \%)$, oxalosis $(4.7 \%)$, other $(20.0 \%)$. At the time of transition, the median age of the patients was 18.9 (range 16.6-23.1) years. $84.7 \%$ of patients were on their first graft, $12.9 \%$ were on their second and $2.4 \%$ were on their third at this point. The immunosuppression at transition was calcineurin inhibitor (CNI) monotherapy in $38.2 \%$, a CNI combination regimen in $20.6 \%$, and a CNI free regimen in $41.2 \%$. Following transition, the estimated 1-, 5- and 10-year patient survival was $98.6 \%, 96.8 \%$ and $91.4 \%$, respectively, and the estimated 1-, 5- and 10-year graft survival was $98.6 \%, 95.0 \%$ and $89.4 \%$.

Conclusion Our results demonstrate that paediatric liver transplant recipients have a better outcome than previously reported in renal transplant recipients after transition. ${ }^{1}$ This may suggest that this group of patients are more tolerant of their graft or that transition was adequately managed.

Competing interests None declared.

\section{REFERENCE}

1. Watson AR. Non-compliance and transfer from paediatric to adult transplant unit. Pediatr Nephrol 2000;14:469-72.

\section{PTU-062 SIGNIFICANT RESPONSE TO LOCAL ABLATIVE BRIDGING TREATMENTS FACILITATES ACCEPTABLE RATES OF SURVIVAL FOLLOWING LIVER TRANSPLANTATION FOR HCC IN A UK CENTRE}

doi:10.1136/gutjnl-2012-302514c.62

R Lochan, ${ }^{*} \mathrm{R}$ Coates, J French, H reeves, B Jaques, S White, D Manas. HPB \& Liver Transplantation Surgery, FRH, Newcastle upon Tyne, UK

Introduction Liver Transplantation (LT) is a well-recognised treatment option for selected patients with hepatocellular carcinoma (HCC). However there is always concern regarding tumour progression while on the waiting list. As yet there is no agreed consensus on how to reduce progression of disease while waiting. UK guidelines recommend local ablative therapy for all HCC patients being considered for LT. We have sought to review this practice and evaluate its benefit.
Methods All consecutive patients with HCC who have undergone LT between 2001 and 2010 were identified from our prospectively maintained database. All patients are discussed at our LT assessment meeting and also at a separate HPB MDT for consideration of bridging treatment. Our imaging protocol includes (1) triple phase CT to assess the number of hyper vascular liver lesions, presence of venous washout, or extra-hepatic disease, (2) MRI to re-characterise any atypical lesions or (3) CEUS for further characterisation. Patients undergo either trans-arterial chemo-embolisation (TACE) and/or percutaneous/laparoscopic radio frequency ablation (RFA) while on the LT waiting list. The response to bridging treatment is assessed and intensive surveillance for disease progression is also undertaken

Results 55 HCC patients underwent LT (M:F=43:12), Childs-Pugh $A(n=9), B(n=30)$ and $C(n=16)$. Bridging treatments were either TACE $n=31$, RFA $n=28$, or both treatments $n=4$. TACE treatments per patient were $1(n=12), 2(n=12), 3(n=6)$ or $4(n=1)$. Six patients did not undergo any form of bridging treatment as they rapidly progressed to $\mathrm{LT}$. The response to bridging treatment was complete $(n=8)$, good $(n=28)$, poor $(n=4)$ or no response $(n=15)$. There were two deaths within 100 post-operative days. At last follow-up, 28 patients had died due to recurrent disease, stable recurrent disease $\mathrm{n}=4$ or disease free $\mathrm{n}=21$. Overall survival [median (95\% CI)] was $62(53-71)$ months. For those with a good response to bridging treatments it was $67(55-79)$ months while for those with poor/no response it was 53 (42-64) months (log-rank $\mathrm{p}=0.059$ ).

Conclusion This study demonstrates the feasibility of various bridging treatments for patients with HCC who await liver transplantation in the UK. In combination with careful patient selection and surveillance acceptable rates of survival can be achieved.

Competing interests None declared.

\section{PTU-063 HIGH RETICULOCYTE COUNT PREDICTS MORTALITY IN PATIENTS AWAITING LIVER TRANSPLANTATION}

doi:10.1136/gutjnl-2012-302514c.63

${ }^{1} \mathrm{R}$ Parker, ${ }^{1} \mathrm{M}$ A Armstrong, ${ }^{1} \mathrm{C}$ Corbett, ${ }^{1}$ I Rowe, ${ }^{1} \mathrm{~B}$ Stephenson, ${ }^{1} \mathrm{D}$ Houlihan, 2J Ferguson. ${ }^{1}$ NIHR Centre for Liver Research and Biomedical Research Unit, Birmingham, UK; ${ }^{2}$ Liver Unit, University Hospitals Birmingham NHS Foundation Trust, Birmingham, UK

Introduction The shortage of donor organs for liver transplantation (LT) makes it essential that organs are allocated to patients with greatest need. There has been increasing interest in haemoglobin as a predictor of LT outcomes. We investigated red cell parameters as predictors of survival after LT assessment.

Methods Data on patients with end-stage liver disease assessed for LT between 2008 and 2010 at University Hospitals Birmingham, UK, were reviewed retrospectively. Kaplan-Meier and Cox regression analysis identified parameters predictive of death on the waiting list. To construct an updated UKELD model including high reticulocyte count (defined as $>80$ ), cases that had not received LT at 12 months were randomly divided into two groups (2:1 ratio) to for model building and testing. Accuracy of the existing and new and models was tested by calculation of c-statistics.

Results Data were collected from 393 patients. Median age was 55 years (range 17-73), 60\% were male. Median UKELD was 56 (44-76). Median follow-up was $18(0-45)$ months. In total 144 (37\%) underwent LT. Abnormal reticulocyte count, seen in 120 patients (31\%), was greatest predictor of death without LT (HR 3.1; $95 \%$ CI 1.7 to 5.6 ), compared to haemoglobin (HR 2.5; 95\% CI 1.3 to 4.5) and MCV (HR 0.6; 95\% CI 0.3 to 1.2). Abnormal reticulocyte count remained a significant predictor of death after adjustment for age, gender and diagnosis (p80; yes $=1, \mathrm{No}=0]$ ). This model had improved predictive accuracy with a c-statistic of 0.79 . 